\title{
A New Way for Spreading Knowledge in Vasculitis
}

\author{
Alexandre Wagner Silva de Souza* \\ Rheumatology Division, Universidade Federal de São Paulo-Unifesp/EPM, Brazil
}

"Corresponding author: Alexandre Wagner Silva de Souza, Rheumatology Division, Universidade Federal de São Paulo-Unifesp/EPM, Brazil, E-mail: alexandre_wagner@uol.com.br

Received date: September 01, 2015; Accepted date: September 03, 2015; Published date: September 06, 2015

Copyright: ( 2015 de Souza W. This is an open-access article distributed under the terms of the Creative Commons Attribution License, which permits unrestricted use, distribution, and reproduction in any medium, provided the original author and source are credited.

\section{Editorial}

In clinical practice, vasculitides are often regarded as extremely rare diseases, that unfortunately sometimes residents and fellows are discouraged to give much attention to these disorders, instead they ought to prioritize more common diseases in their studies. Vasculitis is a group of heterogeneous and protean disorders that pose as a major challenge for internists and physicians from different medical specialties. Diagnosing a vasculitis may also be an important challenge for rheumatologists who are not familiar with this fascinating group of multi organ/system diseases.

The complex nature of systemic vasculitis resides in the fact that different organs and systems may be involved in different combinations while they may be associated with significant accompanying disorders (e.g. hepatitis $\mathrm{C}$, cancer or other systemic autoimmune diseases). Indeed, multiple conditions do mimic manifestations of vasculitis and are usually more prevalent than vasculitis per se [1,2]. Although, the onset of most systemic vasculitides is subacute, the lack of suspicion of a vasculitis in a severely ill patient who actually present a systemic vasculitis is potentially harmful and may result in significant morbidity or even in an increased risk of death [2,3]. It is important to emphasize that attempts should always be made to confirm diagnosis of suspicious cases of vasculitis with appropriate methods that include imaging studies (e.g. angiography for large and medium vessel vasculitis), pathology assessment of affected tissue for small-vessel vasculitis or serologic tests (e.g. antineutrophil cytoplasmic antibodies, anti-C1q antibodies or cryoglobulins) [1,3]. Besides delays in diagnosis, threats for patients with vasculitis also include treating patients with vasculitis who the actual diagnosis has not been established accordingly, improper management (i.e. under-treatment or excessive use of toxic immunosuppressive therapy), misdiagnosis of active disease while a patient presents an infectious complication and inadequate follow-up schedule, to name but a few [3].

In this scenario, Journal of Vasculitis comes as an excellent opportunity for disseminating results from studies on different aspects of vasculitis such as disease manifestations, epidemiology, pathophysiology, diagnostic methods and therapy. Furthermore, interesting clinical images, case reports with unusual manifestations and review articles will assist the learning of physicians from different areas about vasculitis as an additional tool to increase the awareness of these complex disorders. During the last few decades, several advances in research of systemic vasculitis have been made. A universal nomenclature and classification system has been updated based on advances in understanding vasculitis, new outcome measures have been developed to assess disease activity, extension or damage, different studies on epidemiology and genetics have been published including genome wide associated studies (GWAS) [3-5]. Moreover, studies on pathophysiology, especially about alterations in immune response in vasculitis and studies evaluating novel therapeutic agents have been performed in systemic vasculitis, including several randomized controlled clinical trials, mainly multicenter studies [3]. Nonetheless, despite all advances in research of vasculitis to date, there is still a lot of work to be done in order to improve the already established knowledge in vasculitis. Thus, Journal of Vasculitis acts as a unique pathway and as a new possibility for publications in vasculitis and for spreading knowledge about these complex diseases. Hopefully, it will lead to a better understanding, recognition and management of patients with vasculitis. This all may result in further improvements in patient's survival, lessening the burden of complications from accrual damage from disease activity or therapy, resulting in a better quality of life for our patients with vasculitis.

\section{References}

1. Jayne D (2009) The diagnosis of vasculitis. Best Pract Res Clin Rheumatol 23: 445-453.

2. Nataraja A, Mukhtyar C, Hellmich B, Langford C, Luqmani R (2007) Outpatient assessment of systemic vasculitis. Best Pract Res Clin Rheumatol 21: 713-732.

3. Miller A, Chan M, Wiik A, Misbah SA, Luqmani RA (2010) An approach to the diagnosis and management of systemic vasculitis. Clin Exp Immunol 160: 143-160.

4. Jennette JC, Falk RJ, Bacon PA, Basu N, Cid MC, et al. (2013) 2012 revised International Chapel Hill Consensus Conference Nomenclature of Vasculitides. Arthritis Rheum 65: 1-11.

5. Carmona FD, Martín J, González-Gay MA (2015) Genetics of vasculitis. Curr Opin Rheumatol 27: 10-17. 